Digital Press Social Sciences and Humanities

Mer, Forêt, Volcan : défi écologique de l'archipel indonésien dans Mémoires de routes : les iles de la sonde de Thierry Robinet

Andi Mustofa

Proceeding of Conférence internationale sur le français 2018

Joesana Tjahjani, Merry Andriani, Sajarwa, Wening Udasmoro (eds) 


\title{
Mer, Forêt, Volcan : défi écologique de l'archipel indonésien dans Mémoires de routes : les iles de la sonde de Thierry Robinet
}

\author{
Andi Mustofa \\ Département de Français, Universitas Negeri Yogyakarta, Yogyakarta, Indonesia \\ e-mail : anditofa@yahoo.com
}

\section{Résumé}

Le récit de voyage de Thierry Robinet apparaît comme un texte qui propose des regards diversifiés sur la richesse naturelle de l'Indonésie. En parcourant les Mémoires de routes : les îles de la sonde, nous constatons que le questionnement de la relation entre l'homme et la nature reste incontournable. Le travail se propose ainsi de montrer la manière dont la nature indonésienne est représentée ainsi que le regard que l'auteur porte sur les endroits qu'il envisage. Nous examinons par la suite, toujours dans une perspective écocritique, la relation et l'interaction entre l'homme et la nature abordées dans l'œuvre. Le résultat montre que l'auteur caractérise la nature de l'archipel indonésien comme étant une source de vie, une image dynamique voire ambivalente. Le rapport homme-nature se fait dans la pratique de conservation de la nature et la pratique de domination multiforme qui exploite et détruit la nature au profit de l'homme.

\section{Mots-clés}

écologie, homme-nature, écocritique, Indonésie

\begin{abstract}
The travel story of Thierry Robinet appears as a text that offers diversified looks on the ecology of Indonesia as having a great natural diversity. In navigating the Mémoires de routes: les îles de la sonde, we see that questioning the relationship between human beings and nature is unavoidable. The work thus proposes to show how the author perceives and characterizes the nature of Indonesian archipelago. We look at later, always in an ecocritique perspective, the relationship and interaction between human beings and nature in the work. The result shows that the author, as a Westerner, seems to characterize the nature Indonesian archipelago as being dynamic, wild, dangerous, and full of mysteries, wealth and wonders. The relation human-nature happens in the practice of nature conservation and the practice of multiform domination which exploits and destroys nature for the benefit of human.
\end{abstract}

\section{Keywords}

ecology, human-nature, ecocritique, Indonesia

\section{Introduction}

« Où est la place de l'homme-nature aujourd'hui dans le tourbillon infernal de notre vie, où les grandes zones vertes de notre planisphère régressent de jour en jour devant l'avancée humaine ? " (Robinet, 2016, p. 117)

Dès la première partie de ses mémoires de routes, Thierry Robinet est fasciné par les traditions, les histoires et surtout les paysages naturels rencontrés durant ses explorations en Asie, plus particulièrement en Indonésie. Définie comme étant « tout ce qui existe sur la Terre hors de l'homme et des œuvres de l'homme » (Rey, 2013, p. 994), la nature est mise en accent dans son récit de voyage intitulé Mémoires de routes : Les îles de la Sonde qui a été écrit selon ses expériences du voyage d'une quarantaine d'années dans le plus grand archipel au monde. Le voyage de Thierry Robinet est guidé par le 
désir d'aventure pour rencontrer avec ceux qu'il ne connait pas et en même temps rencontrer avec soimême (Robinet, 2016). À l'âge de seize ans, Thierry Robinet, fils d'un ancien professionnel de football, décide de commencer son premier voyage en Europe de l'Est en 1969 et en Orient d'Asie Mineur jusqu'en 1972. Son intérêt de découvrir le monde est dû à son père qui lui proposait une activité chaque weekend avec les cartes de géographies : " un concours alphabétique des continents, pays et villes mondiales » (Robinet, 2016, p. 21). Son esprit d'aventure devient plus grand lorsqu'il décroche son diplôme d'agent de voyages à Chambéry, en Savoie. Il le décrit : « une nouvelle ère s'ouvrait, le départ pour de nombreux voyages lointains aux quatre coins de la planète, et surtout 'aller là où les autres n'allaient pas' » (Robinet, 2016, p. 75).

Dans ce travail, nous tenterons d'analyser la manière dont la nature indonésienne est représentée ainsi que le regard que l'auteur porte sur les endroits qu'il envisage. Il semblerait que l'auteur avec son point de vue occidental caractérise la nature de l'archipel indonésien comme étant dynamique, sauvage, dangereuse et plein de richesses. Ces derniers aspects soulignés montreraient le rapport créé par l'auteur avec la nature ainsi que celui des autochtones.

Les défis écologiques se concentrent sur la planète, la nature et des sociétés qui en font partie. En parcourant les Mémoires de routes : Les îles de la Sonde, nous nous rendons compte que le questionnement de la relation entre l'homme et la nature - la forêt, la mer et le volcan - reste incontournable. Ainsi, nous tâcherons de montrer comment l'auteur révèle le rapport de l'homme avec la nature de l'archipel indonésien. Il semblerait que les autochtones - ceux qui sont nés et vivent depuis leur naissance dans la nature décrite par l'auteur - ont une relation différente avec la nature par rapport aux non autochtones qui viennent d'entrer en contact avec ce milieu naturel raconté dans l'œuvre.

Pour mener à bien notre recherche, nous nous appuierons sur le concept écrocritique. Ce concept commence à entrer dans le domaine littéraire au milieu des années soixante-dix. Pourtant, ce concept ne se développe qu'au début des années quatre-vingt-douze grâce à l'Association for the Study in Literature and Environment (ASLE) aux États-Unis qui fait naître plusieurs textes sur l'écocritique et ceux-ci essaient, à travers une approche interdisciplinaire : de l'écologie à la littérature en passant par la géographie et la biologie, de répondre à la crise de l'environnement (ASLE, https://www.asle.org/ consulté le 15 août 2018).

L'écrocritique souligne la relation dynamique entre la littérature et l'environnement naturel. Ceci correspond au concept de l'écocritique proposé par Cheryll Glotfelty dans The Ecocriticism Reader.

"What then is ecocriticism? Simply put, ecocriticism is the study of the relationship between literature and the physical environment. Just as feminist criticism examines language and literature from a gender-conscious perspective, and Marxist criticism brings an awareness of modes of production and economic class to its reading of texts, ecocriticism takes an earth-centered approach to literary studies » (Glotfelty, 1996, p. xviii)

L'écocritique a pour but de chercher la réponse aux problèmes écologiques, de faire naître la vision écologique de l'homme et de comprendre la relation symbiotique et dynamique entre des êtres humains et la nature. Ainsi, Cheryll Glotfelty propose un modèle pour s'approcher le texte écocritique. D'abord, il s'agit d'analyser la manière dont la nature est représentée dans le texte. La nature décrite dans le texte englobe quelques éléments tels que les animaux, les montagnes et les déserts. Ensuite, il s'agit d'analyser des influences de l'environnement afin de comprendre la relation créée entre l'homme et l'environnement dans le texte. Enfin, il s'agit d'examiner la construction de l'humain dans son environnement (Glotfelty, 1996).

\section{Méthode}

Ce présent travail est une recherche descriptive-qualitative en utilisant comme un objet de recherche le récit de voyage de Thierry Robinet intitulé Mémoires de routes : Les îles de la Sonde publié en 2016 par la maison d'édition Adalta. L'analyse de notre travail se base sur le concept écocritique comme étant un point de départ pour analyser l'œuvre choisie. Ceci permet à travers une analyse critique de comprendre le rapport dynamique et symbiotique établi entre l'homme et la nature, de connaître le cas singulier de l'homme dans sa manière de voir le monde et de créer la relation à l'autre et à lui-même.

L'analyse critique se fait en deux étapes. La première étape se concentre sur la représentation de la nature de l'archipel indonésien décrite par l'auteur lors de son voyage en Indonésie. La description de l'auteur montre une représentation qui aide à la compréhension de la nature et de l'homme qui en fait partie. La deuxième étape se fait par l'observation de la perception de l'homme face à des milieux naturels. Les pratiques faites par les autochtones et les non autochtones dans la nature sont ensuite 
observés ainsi que les impacts de ces activités humaines sur la nature et la population afin de voir la relation l'homme-nature et la construction de l'être humain.

\section{Résultats et discussion}

\subsection{La nature indonésienne : une mère nourricière et une mère meurtrière}

"L'Indonésie est un pays d'une richesse considérable que ce soit sur et sous la mer, les terres, les jungles et les volcans. Les milliers d'espèces endémiques à toutes ces régions dispersées de part et d'autre de l'équateur sont le trésor de l'Indonésie » (Robinet, 2016, p. 127)

La nature de l'Indonésie propose une grande diversité grâce à la position géographique qui permet d'avoir un climat tropical. Au moment du voyage à Bornéo, l'auteur découvre un petit groupe Dayak de chasseurs-cueilleurs: les Punans. L'auteur le considère comme "l'un des groupes ethniques les plus pacifiques et paisibles de notre Terre » (Robinet, 2016, p. 115). Il existe plus ou moins neuf mille Punans qui survivent et dépendent de la jungle de Sarawak et de Kalimantan, autour de la région des fleuves Kayan et Rajang. L'auteur le décrit : « la forêt était et est encore aujourd'hui leur habitat de prédilection » (Robinet, 2016, p. 115).

La découverte de l'auteur sur la dépendance des Punans à la forêt tropicale va plus loin lorsqu'il fait le contact avec ce petit groupe dayak de manière plus profonde. L'auteur le raconte : « pendant des siècles, les arbres et plantes de la forêt leur ont servi de cabinet médical, outils et abris » (Robinet, 2016, p.116). De cette citation, la forêt est pour les Punans le centre de tout. Autrement dit, la forêt est une source de vie et un élément essentiel dans la vie. La relation établie entre les Punans et la forêt s'inscrit dans un contexte sanitaire et social. Ainsi, la forêt est la vie et les Punans en font partie. La forêt apporte tout ce dont les Punans ont besoin.

Le rapport particulier de la nature est décrit lorsque l'auteur découvre la mer dans laquelle le peuple Bajau vit. Il le décrit : " un groupe très spécial [...] composé des nomades de la mer ; le peuple Bajau » (Robinet, 2016, p.119). Comme les Punans, les Bajau dépendent de la nature. Ce peuple subsiste dans les estuaires proches des mangroves et eaux dormantes des lagons

«Ils ont suivi les côtes nord de Sulawesi pour s'installer dans de petites criques et des îlots où ils ont pu vivre en paix et à l'abri des regards. Leur migration dès le XVIII e siècle les a vus aller jusque sur les côtes de Sulawesi, Bornéo, Sumatra, Florès, Komodo et le nord de l'Australie » (Robinet, 2016, p. 119)

La mer est centre de tout pour les Bajau. Elle est un symbole maternel. L'attrait et le besoin de la mer sont décrits par l'auteur : « [Les Bajau] peuples de la mer, nomades de la mer, damnés de la mer, gitans de la mer. Que de qualificatifs empruntés pour ces hommes paisibles qui ont la mer pour unique ressource » (Robinet, 2016, p. 119). De cette citation, la mer apporte une sorte de protection. Celle-ci ressemble à l'image de la mère nourricière.

Le rapport intime avec la nature permet de définir le peuple qui en fait partie. Dans le cas du peuple Bajau, le mode de vie chez les Bajau est influencé par la mer comme étant un élément principal dans la vie. De plus, l'exigence de la mer est considérée plus essentielle que le cycle quotidien du temps. L'auteur le remarque :

« Le temps ne compte pas pour les Bajau [...] La vie se règle sur les marées, les courants et les mouvements saisonniers du poisson. Le cycle quotidien de la nuit et du jour, du lever et du coucher de soleil, n'est que secondaire comparé aux exigences de la mer » (Robinet, 2016, p. 120-121)

L'amour pour la mer qui joue le rôle le plus important dans le cycle de la vie chez les Bajau crée une dépendance extrême, voire une soumission à la mer. Si le peuple Bajau meurt, sa volonté d'être en contact avec la mer ne s'arrête pas. La mort n'est pas la raison d'être loin de la mer. A contrario, le lien avec la mer doit continuer à être établi.

« Tout le paradoxe est là lorsqu'un homme [Bajau] meurt [...] Son bateau est démonté et les planches lui serviront de cercueil. Il sera enterré sur la plage, le regard tourné vers la mer, et il va de son tombeau scruter le grand large, veiller magiquement sur les leppaset leurs familles. Symboliquement, il continuera à naviguer » (Robinet, 2016, p. 123-124)

Bien que le corps soit enterré, l'esprit doit être toujours vers la mer. De ce fait, il semble que les Bajau ont une devise : c'est à la mer que nous appartenons et c'est vers la mer que nous retournerons. Pour eux, la mer est pour la vie et pour la mort.

Le besoin de la nature se voit également dans la description de l'auteur lors de la découverte des volcans indonésiens. Il s'agit de Krakatau et Merapi qui selon l'auteur sont considérés ambivalents. Il est source de la vie et en même temps de la mort. 
« Les pêcheurs de Sumatra visitent au quotidien pour son pourtour riche en poissons, ses pythons énormes qu'ils capturent de nuit sur l'île de Rakata pour les revendre aux gens des côtes, ainsi que les crabes aux larges princes qui, les jours de pleine lune, s'amassent par centaines sur les minces bandes de sable gris » (Robinet, 2016, p. 251)

Le volcan Krakatau est considéré comme étant une mère nourricière. Il est une figure pour se ressourcer et se nourrir. Cependant, son image peut devenir " un monstre sacré au milieu de la mer » (Robinet, 2016, p. 251). L'auteur le remarque : « en 1680, une violente éruption accompagnée d'un raz-demarée provoque de gros dégâts sur Sumatra et Java [...] De 1680 à 1883, il n'y a aucune activité » (Robinet, 2016, p. 246). De cette citation, la nature donne une autre réalité. Celle-ci présente également une source de danger. Ainsi, la nature est comme une mère meurtrière : « si le Merapi explosait [...] le véritable danger, ce sont ses coulées de boue, les lahars, mélange de cendres, de blocs de rochers explosés et d'eau crachés par le volcan, que les pluies diluviennes de la région entrainent en coulées dévastatrices » (Robinet, 2016, p. 253).

Le peuple autour du volcan se rend compte du danger de l'activité volcanique de Krakatau et de Merapi. Pourtant, ils n'ont pas tendance à se renfermer dans un état d'esprit d'insécurité. Au contraire, ils considèrent le danger de l'explosion du volcan comme une source de vie, c'est-à-dire que cette explosion est un moyen de Dieu pour envoyer son amour et ses grandes bénédictions pour le peuple autour du volcan.

«Pour celui qui a vu de nombreuses fois les terribles coulées pyroclastiques ou de lahars du Merapi, chaque soubresaut du volcan est d'un intérêt capital pour la survie des populations alentours [...] Pour d'autres, les centaines d'ouvriers des carrières du lit de la rivière Krasak, vers Jurangjero, c'est [les blocs de pierre] une manne envoyée par les dieux. Les camions et les hommes attendent les blocs de rochers lâchés du sommet du dôme qui serviront pour la construction de routes ou de maisons dans la vallée toute proche » (Robinet, 2016, p. 258-259)

Ainsi, le volcan est considéré comme étant une image dynamique voire ambivalente. D'un côté, il menace la vie et fait peur. De l'autre côté, il donne la vie et enrichit le peuple alentour.

La nature joue son rôle important à travers la force de nourrir et de modifier la condition soit physique soit psychologique de l'être humain. Dans le cas singulier de Thierry Robinet, la forêt et la mer présentent une véritable source de vie, sans aucun danger. Ceci est différent dans le cas des volcans Krakatau et Merapi qui sont un exemple d'ambiguïté. Les volcans sont considérés comme étant une figure de mère nourricière et à la fois meurtrière.

\subsection{La sacralisation de la nature}

Dans ce chapitre, nous abordons la question du rapport et de la complexité de la relation créée par l'homme avec la nature. L'action de l'être humain sur l'environnement correspond à ses besoins. Celle-ci est illustrée par l'auteur sous forme du respect des Punans à la nature puisque « les esprits des arbres sont leur raison de vivre » (Robinet, 2016, p. 116). Ainsi, garder la forêt veut dire garder l'existence du groupe puisque la vie des Punans est étroitement liée à la forêt. Lorsque cette harmonie est rompue, ceci affecte et dérange le rythme chez les Punans.

Comme nous avons montré précédemment, la nature pour les Punans est une source primaire dans leur vie. Pour eux, toutes les activités doivent être mesurées, dans le sens que celles-ci n'entrainent une rupture de l'harmonie ni détruisent la forêt comme étant la source essentielle de vie. Exploiter la forêt se fait dans le respect de la nature et de la population. Ainsi, la stratégie et la pratique de conservation sont faites afin de pouvoir préserver l'équilibre de la nature et de l'harmonie de la vie.

Une conscience écologique est également présente chez les Bajau. Comme nous avons montré dans le chapitre précédent, la mer pour les Bajau joue un rôle important comme étant une source qui offre de nombreux avantages. La mer est la mère nourricière. La conservation de la mer se fait dans l'utilisation de la leppapour pêcher. La leppaest une pirogue en bois très équilibrée et celle-ci peut soutenir de grands vents. Les Bajau n'exercent pas avec cette pirogue la pêche à la dynamite qui tue le biote marin. De plus, la cérémonie traditionnelle se fait afin de remercier les gardiens de la mer et garder l'équilibre et l'harmonie entre les Bajau et la mer : « ils accordent une large place aux génies du vent et des eaux, aux cérémonies de la vie et de la mort, aux présages et aux superstitions » (Robinet, 2016, p. 123).

La même stratégie se fait par le peuple autour du volcan Merapi. Ils font un rite traditionnel pour vivre en harmonie avec le volcan. L'auteur le remarque :

«Marijan a toujours un bon mot pour eux [les villageois], les apaise et leur dit de ne pas avoir peur. « Quand je veux contacter le génie du volcan, je jeûne et me recueille pendant plusieurs jours. Au bout de quelques temps, le Merapi m'apparaît et il me parle de son humeur. Quand il se met à gronder, une éruption risque de commencer. Aussi je discute avec lui inlassablement pour le calmer, l'apaise avec mes 
offrandes de riz, de fleurs et plusieurs poulets, les plus beaux du village. Le génie qui habite dans le cratère a besoin de se nourrir » (Robinet, 2016, p. 255-256)

La mer et le volcan sont l'objet de croyances pour les Bajau et le peuple autour de la montagne de feu. La clé pour vivre en harmonie avec eux n'est pas faite que dans la pratique de conservation, mais aussi dans le contact avec les divinités. Ainsi, le lien entre l'homme et la nature se crée à travers le rapport physique avec la nature ainsi que le rapport non physique avec l'esprit dans le monde de l'au-delà

Une relation profonde et sacrée est établie entre la nature et les sociétés de la forêt, de la mer et du volcan. Ce rapport intime se crée dans le respect de la nature, voire dans la soumission de l'homme à la nature. Ainsi, la nature reste 'supérieure' par rapport à l'homme. Toutefois, les autochtones, bien qu'ils soient 'inférieurs', peuvent prendre l'avantage d'exploiter la nature de manière durable et responsable.

\subsection{La désacralisation de la nature}

La soumission à la nature est liée au sentiment de peur chez l'homme de ne plus recevoir les ressources de vie au contact de la forêt, de la mer et du volcan. Ainsi, le respect de la nature se crée. Pourtant, il semble que le sentiment de peur lié au respect de la nature n'est pas présent chez les non autochtones. L'auteur le décrit :

"Quelques groupuscules vont et viennent de chaque cote de la frontière pour échapper à la pression grandissante des immenses sociétés du bois, qui au quotidien coupent l'une des plus grandes réserves de la biosphère. Les bois précieux rapportent des milliards de dollars, et sur les sols laissés déserts, on plantera le palmier à huile pour une demande considérable de la part des nouveaux pays riches ou en voie de développement » (Robinet, 2016, p. 115)

Nous remarquons dans les cultures chez les non autochtones, l'intérêt d'entrer en contact avec la nature a pour but de traiter la nature sans limites et de l'exploiter de manière excessive au profit de l'homme. Cet acte provoque la dégradation de l'environnement sous forme de plusieurs phénomènes tels que la pollution, l'apparition de nouvelles maladies, la perte des espèces animaux et végétales ainsi que des ressources naturelles. L'auteur métaphorise cette dégradation de la forêt chez les Punans « comme le bois qui se consume dans le feu » (Robinet, 2016, p. 117).

Le manque de respect de la nature est aussi présent dans la mer. Ceci est montré dans la technique de pêche pratiquée par les non autochtones qui tue le biote marin : «ils [les bateaux-usines en provenance de Chine ou de Taiwan] ne sont pas Bajau, ceux qui partent à la chasse au napoléon ou au keraputikusà la chair si savoureuse et que les restaurants de Hong Kong se disputent à prix d'or » (Robinet, 2016, p. 124). Cette pratique de pêche se fait par une explosion sous-marine qui très rapidement tue des poissons. Ceci augmente la perte irréparable de la vie marine puisque de petits poissons ne verront pas l'âge adulte.

L'exploitation de la nature sans responsable se voit comme étant une manière de l'homme pour dominer l'existence de la nature. Ce fait est dû aux non autochtones qui se sentent séparés de ce milieu naturel puisque ceux-ci ne font pas partie, dans le cas de Thiery Robinet, de la forêt et de la mer. Par conséquent, ils ne sont pas soumis à la loi de la nature. Ils se considèrent supérieurs et se permettent d'agir contre les lois de la nature à travers la pratique d'exploitation abusive des ressources naturelles.

\section{Conclusion}

La nature - la forêt, la mer et le volcan - est pour les autochtones une source de vie. Elle est dynamique voire ambivalente - quelques fois considérée comme étant une mère nourricière et meurtrière - et celleci devient une figure primordiale pour se ressourcer et se nourrir. L'amour pour la nature chez les autochtones crée une relation intime, une dépendance voire une soumission à la nature qui encourage le respect et la conscience de maintenir l'harmonie de la vie. Ainsi, un rapport sacré se produit entre la nature et les autochtones de la forêt, de la mer et du volcan.

Les non autochtones se considèrent supérieurs par rapport à la nature et se permettent d'agir contre les lois de la nature. La nature est ainsi désacralisée sans respect par la pratique d'exploitation abusive des ressources naturelles. Par conséquent, celle-ci entraine la dégradation de l'environnement.

Pour conclure, le récit de voyage de Thierry Robinet sensibilise à l'importance de la nature pour l'existence de l'être humain. L'équilibre de la nature est nécessaire à l'avenir de l'homme pour vivre en harmonie. Comme la nature est sacrée et l'être humain en fait partie, le respect et la protection de la nature doivent être encouragés dès maintenant à travers l'exploitation de la nature de manière durable et responsable pour l'avenir de l'humanité. 


\section{Références}

Association for the Study of Literature and Environment. (2018). Association for the Study of Literature and Environment. Retrieved from https://www.asle.org/

Glotfelty, C. (1996). Literary Studies in an Age of Environmental Crisis. In Cheryll. Glotfelty \& H. Fromm (Eds.), The Ecocriticism Reader: Landmarks in Literary Ecology (pp. xv-xxxvii). Athens: The University of Georgia Press.

Rey, A. (2013). Le Petit Robert Micro. Paris: Le Robert.

Robinet, T. (2016). Mémoires de routes : Les Iles de La Sonde. Leipzig: Adalta. 